\title{
THE CARTAN MATRIX AS AN INDICATOR OF FINITE GLOBAL DIMENSION FOR ARTINIAN RINGS
}

\author{
W. D. BURGESS ${ }^{1}$, K. R. FULLER, E. R. VOSS AND B. ZIMMERMANN - HUISGEN \\ ABSTRACT. A left serial ring has finite global dimension if and only if its Cartan \\ matrix has determinant equal to 1 .
}

1. Introduction. A slight modification of a counting argument given by Eilenberg (see [2, Proposition 21; 13, Proposition 1.1]) three decades ago shows that the determinant of the Cartan matrix $C$ of a left artinian ring $R$ of finite global dimension is always 1 or -1 . No instances are known in which the value -1 is attained. In a recent paper, Zacharia [14] proved that the determinant is, in fact, 1 whenever $\operatorname{gl} \operatorname{dim} R \leqslant 2$. We continue this line by establishing $\operatorname{det} C=1$ for left serial rings of finite global dimension. What is more surprising is the fact that, conversely, det $C=1$ guarantees finite global dimension for any left serial ring. This provides a simple finitary procedure for determining whether a left serial ring is of finite dimension. The latter implication does not extend to arbitrary left artinian rings; we exhibit an example which deviates "as slightly as possible" from being left serial, but which nevertheless combines det $C=1$ with infinite global dimension.

The phenomenon just described already occurs for artinian rings of Loewy length 2. On the other hand, for Loewy length 2, the Cartan matrix as a whole still reflects whether or not the global dimension is finite; this was observed by Jans and Nakayama [8, Proposition 10]. It is natural to look at Loewy length 3 next. Here, knowledge of the Cartan matrix no longer suffices for the distinction between finite and infinite global dimension; in fact, we construct two artinian rings of Loewy length 3 with identical Cartan matrices, one of which has finite, the other infinite, global dimension.

It is still open whether finite global dimension implies $\operatorname{det} C=1$ for all left artinian rings. The positive answers in the special cases $\mathrm{gl} \operatorname{dim} R \leqslant 2, R$ left serial, $R$ of Loewy length $\leqslant 2$ are based on the existence of a simple left module of projective dimension $\leqslant 1$ (discarding the corresponding primitive idempotent gives rise to a finite induction). Our final disillusioning example demonstrates that this road will not lead to a positive decision in the general setting.

Throughout, $R$ will denote a left artinian ring with Jacobson radical $J$, and $\left\{e_{1}, \ldots, e_{n}\right\}$ will be a complete orthogonal set of pairwise nonisomorphic primitive idempotents. In particular, the isomorphism types of the simple modules (module

Received by the editors September 6, 1984.

1980 Mathematics Subject Classification. Primary 16A03, 16A35.

${ }^{1}$ The work of the first author was partially supported by grant A7539 of the NSREC and was done while he was enjoying the hospitality of the University of Iowa.

(C)1985 American Mathematical Society $0002-9939 / 85 \$ 1.00+\$ .25$ per page 
stands for left module unless otherwise indicated) are $S_{1}, \ldots, S_{n}$ where $S_{i}=R e_{i} / J e_{i}$. Recall that the Cartan matrix $C=C\left({ }_{R} R\right)$ is the $n \times n$ matrix with integral entries $c_{i j}$ which are defined as follows: $c_{i j}$ is the number of copies of the simple module $S_{i}$ appearing in a composition series for $R e_{j}$. Clearly, the ring $R$ has the same Cartan matrix as its basic ring. Moreover, if $R=R_{1} \times R_{2}$ is a ring decomposition, $C$ is a block diagonal matrix with blocks $C\left(R_{1}\right)$ and $C\left(R_{2}\right)$. Hence, in the sequel we may always assume that $R$ is basic and indecomposable.

2. Left serial rings. Recall that a left artinian ring $R$ is called left serial if each of the indecomposable projective modules $R e_{i}$ is uniserial, that is, contains a unique composition series. Serial rings are rings satisfying this condition on both sides.

Examples of serial rings can, for instance, be found in [4] and [11]; for examples of left serial rings which are not serial, see [9].

The sequence $R e_{1}, \ldots, R e_{n}$ is called a (left) Kupisch series if $R e_{i}$ is a projective cover of $J e_{i+1}$ for $1 \leqslant i \leqslant n-1$, and $R e_{n}$ is a projective cover for $J e_{1}$ or $J e_{1}=0$. By [10] such series exist whenever $R$ is serial; if $R$ is QF also, then all $R e_{i}$ have the same composition length [11, Theorem 19]. Thus, the following computation yields, in particular, the Cartan matrices of QF serial rings.

LEMMA 1. Let $R$ be left serial, and suppose that $R$ has a Kupisch series $R e_{1}, \ldots, R e_{n}$, all of whose members have the same composition length $m$. Write $m=$ an $+r$ with $0 \leqslant r<n$. Then $C$ has the following form:

$$
c_{i j}= \begin{cases}a+1 & \text { if } 0 \leqslant j-i<r \text { or } n-r<i-j \leqslant n, \\ a & \text { otherwise. }\end{cases}
$$

(In other words, for $r>0$, the matrix $C$ has entries $a+1$ on the main diagonal, on the next $r-1$ superdiagonals, and on the subdiagonals from $n-r+2$ down, and the remaining entries are all $a$.)

ProOF. The sequence of composition factors of $R e_{j}$ is $S_{j}, S_{\overline{j-1}}, \ldots, S_{\overline{j-(n-1)}}, S_{j}$, $S_{\overline{j-1}}, \ldots,(\bar{k}$ stands for the least positive remainder of $k$ modulo $n$ ); it continues for $m$ terms. Thus, there are $a+1$ copies of the first $r$ candidates in the list and $a$ copies of the others.

The matrices described in Lemma 1 are types of circulant matrices. We record a special case of [1, Problem 27, p. 81] as

LEMMA 2. Let $C$ be a matrix as in the previous lemma. Then

$$
\operatorname{det} C= \begin{cases}m & \text { if } \operatorname{gcd}(m, n)=1, \\ 0 & \text { if } \operatorname{gcd}(m, n) \neq 1\end{cases}
$$

LEMMA 3. If $R$ is left serial with a simple module of finite projective dimension, then $R$ has a simple module of projective dimension $\leqslant 1$.

Proof. Either $R$ has a simple projective module, or the minimal projective resolution of a simple module of finite projective dimension $\geqslant 1$ provides us with a proper monomorphism $O \rightarrow R e_{i} \rightarrow R e_{j}$ whose image is $J^{m} e_{j}$, say. In the latter case, if $R e_{k} \stackrel{g}{\rightarrow} J^{m-1} e_{j} \rightarrow 0$ is a projective cover, $g$ induces a split epimorphism $J e_{k} \rightarrow J^{m} e_{j}$ $\cong R e_{i}$. But $J e_{k}$ is indecomposable, and hence this is an isomorphism. It follows that $R e_{k} / J e_{k}$ has projective dimension 1. 
One direction of the equivalence in the next lemma is due to Zacharia [14, Lemma 2]; the inequality appears in Gustafson [7] with the added hypothesis that $R$ be serial and $\operatorname{gl} \operatorname{dim} R<\infty$. In establishing the bridge between Cartan matrix and global dimension, we will use it to successively reduce the number of primitive idempotents.

Lemma 4. Let $R$ be left serial and $e=1-e_{1}$. Provided that $\mathrm{p} \operatorname{dim} S_{1} \leqslant 1$, the left global dimension of $R$ is finite if and only if the left global dimension of eRe is finite, and $1 \mathrm{gl} \operatorname{dim} R \leqslant 1 \mathrm{gl} \operatorname{dim} e R e+2$.

Proof. First suppose that $1 \mathrm{gl} \operatorname{dim} e R e$ is finite.

If $\mathrm{p} \operatorname{dim} S_{1}=0$, we have $J e_{1}=0$; if $\mathrm{p} \operatorname{dim} S_{1}=1$, then $J e_{1} / J^{2} e_{1} \not S_{1}$. In both cases we find $\operatorname{Ext}^{1}\left(S_{1}, S_{1}\right)=0$.

Now let $i \neq 1$ and $\mathrm{p} \operatorname{dim}_{e R e} e S_{i}=m$. In order to verify that $\mathrm{p} \operatorname{dim} S_{i} \leqslant m+2$, consider a projective resolution

$$
\cdots \rightarrow P_{k} \stackrel{f_{k}}{\rightarrow} P_{k-1} \rightarrow \cdots \rightarrow P_{1} \stackrel{f_{1}}{\rightarrow} P_{0} \stackrel{f_{0}}{\rightarrow} S_{i} \rightarrow 0
$$

of $S_{i}$, where all the $P_{k}$ are indecomposable. As Zacharia [14, p. 355] observed (note that his argument for $\mathrm{p} \operatorname{dim} S_{1}=1$ works equally well for $\mathrm{p} \operatorname{dim} S_{1}=0$ ), the sequence

$$
\cdots \rightarrow e P_{k} \rightarrow e P_{k-1} \rightarrow \cdots \rightarrow e P_{1} \rightarrow e P_{0} \rightarrow e S_{i} \rightarrow 0
$$

is then an $e R e$-projective resolution of the $e R e$-module $e S_{i}$. In particular, $f_{m}\left(e P_{m}\right)$ is projective and nonzero. Since $e P_{m}$ is indecomposable, we infer that $\left.f_{m}\right|_{e P_{m}}$ is monic. Set $T=\operatorname{Soc} P_{m}$.

If $T \not S_{1}$, we have $0 \neq f_{m}(e T) \subset f_{m}(T)$, whence $f_{m}: P_{m} \rightarrow P_{m-1}$ is also monic, and $\mathrm{p} \operatorname{dim} S_{i} \leqslant m$.

If $T \cong S_{1}$, we invoke $\operatorname{Ext}^{1}\left(S_{1}, S_{1}\right)=0$ to obtain a submodule $L$ of $P_{m}$, namely $L=\operatorname{Soc}^{2} P_{m}$, such that $L / T \not S_{1}$. From $0 \neq f_{m}(e L) \subseteq f_{m}(L)$, it follows that $L \nsubseteq$ $\operatorname{ker} f_{m}$ and, consequently, $\operatorname{ker} f_{m} \subseteq L$. From $\operatorname{ker} f_{m} \neq L$ we conclude further that $\operatorname{ker} f_{m}$ equals either $T$ or 0 . In the latter case we have $\mathrm{p} \operatorname{dim} S_{i} \leqslant m$ as above. In the former we obtain an exact sequence

$$
0 \rightarrow J e_{1} \rightarrow R e_{1} \rightarrow P_{m} \stackrel{f_{m}}{\rightarrow} \cdots \rightarrow P_{0} \rightarrow S_{i} \rightarrow 0
$$

which, in view of the projectivity of $J e_{1}$, yields p $\operatorname{dim} S_{i} \leqslant m+2$.

Conversely, finiteness of $1 \mathrm{gl} \operatorname{dim} R$ implies finiteness of $1 \mathrm{gl} \operatorname{dim} e R e$, since, as we have already remarked, multiplication of an $R$-projective resolution of a simple $S_{i}$ $(i \geqslant 2)$ by $e$ results in an $e R e$-projective resolution of $e S_{i}$.

The last of our preparatory lemmas is due to Zacharia [14, Theorem B]. We include a particularly simple argument.

LemMA 5. Suppose that the projective dimension of $S_{1}=R e_{1} / J e_{1}$ is $\leqslant 1$ and set $e=1-e_{1}$. Then $\operatorname{det} C(e R e)=\operatorname{det} C(R)$.

Proof. By hypothesis,

$$
J e_{1}=\left(R e_{2}\right)^{m_{2}} \oplus \cdots \oplus\left(R e_{n}\right)^{m_{n}} \text { with } m_{i} \geqslant 0 .
$$


Denoting the $j$ th column of the Cartan matrix $C$ of $R$ by

$$
c_{j}=\left(\begin{array}{c}
c_{1 j} \\
\vdots \\
c_{n j}
\end{array}\right)
$$

we obtain

$$
c_{1}=\left(\begin{array}{c}
1 \\
0 \\
\vdots \\
0
\end{array}\right)+\sum_{j=2}^{n} m_{j} c_{j}
$$

Thus, subtraction of $m_{j}$ times the $j$ th column from the first column for $j=2, \ldots, n$ yields the matrix

$$
\left(\begin{array}{cccc}
1 & c_{12} & \cdots & c_{1 n} \\
0 & c_{22} & \cdots & c_{2 n} \\
\vdots & \vdots & & \vdots \\
0 & c_{n 2} & \cdots & c_{n n}
\end{array}\right)
$$

and consequently,

$$
\operatorname{det} C=\operatorname{det}\left(\begin{array}{ccc}
c_{22} & \cdots & c_{2 n} \\
\vdots & & \vdots \\
c_{n 2} & \cdots & c_{n n}
\end{array}\right)
$$

But the latter matrix is the Cartan matrix of $e R e$.

Recall that the quiver of a left artinian ring $R$ is a directed graph with vertices $e_{1}, \ldots, e_{n}$ and precisely $r$ arrows $e_{i} \rightarrow e_{j}$ if $J e_{i} / J^{2} e_{i}$ contains $r$ copies of $S_{j}$ (see, for example, [6, pp. 88, 119]). Since our ring is indecomposable, its quiver is connected as an undirected graph.

In the case of a left serial ring, the quiver has a particularly tractable form: it is either a rooted tree or a graph obtained from a rooted tree by replacing its root by an oriented cycle. (Here we go against the usual convention by having the arrows point towards the root).

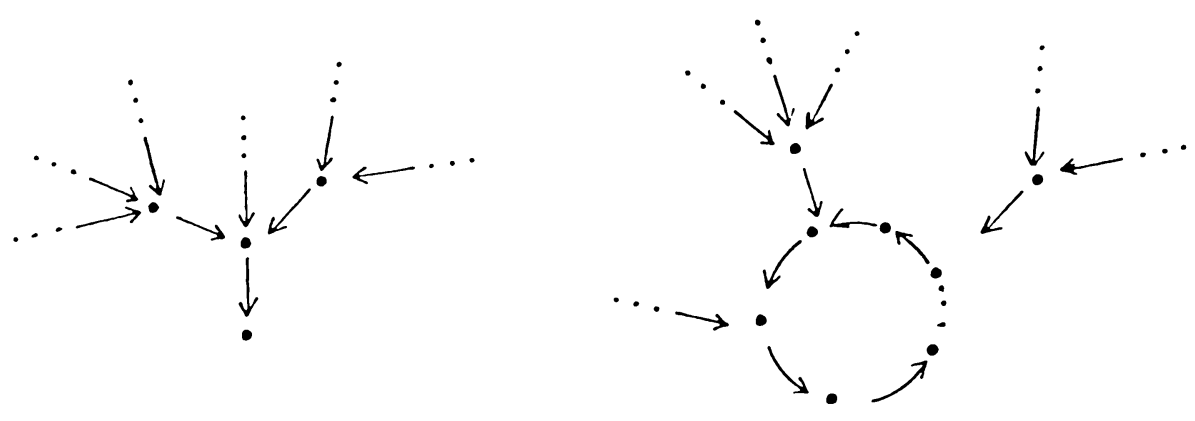


To see this, we first note that $R$ is left serial if and only if at most one arrow emanates from any given vertex. As in [5], a quasi-order can be introduced on the set of vertices via $e_{i}<e_{j}$ if $i=j$ or if there is an oriented path from $e_{j}$ to $e_{i}$. Let $e_{1}$ be a minimal element with respect to this quasi-order, and consider the set $S=\left\{e_{i} \mid e_{i} \leqslant\right.$ $\left.e_{1}\right\}$; together with the arrows between these vertices, $S$ either forms an oriented cycle or a loop 0 , or else a graph consisting just of one point $\bullet$. Since there are never two arrows leaving a given vertex (and since the underlying undirected graph is connected), any vertex belonging to a cycle or a loop lies in $S$.

Now weight each vertex $e_{i}$ by the composition length $c_{i}$ of $R e_{i}$. Then the weights along an oriented path in the quiver of a left serial ring $R$ behave like the admissible sequences of serial rings [4]. More precisely, if $e_{i} \rightarrow e_{j}$ is an arrow in the quiver, that is, $R e_{j}$ is a projective cover of $J e_{i}$, then clearly $c_{j} \geqslant c_{i}-1$, and $c_{j}=c_{i}-1$ if and only if $\mathrm{p} \operatorname{dim} R e_{i} / J e_{i}=1$.

The following remark evokes the situation described in Lemma 1. If $e_{1}=e_{i_{1}} \rightarrow e_{i_{2}}$ $\rightarrow \cdots \rightarrow e_{i_{k}} \rightarrow e_{1}$ is an oriented cycle, then $R e_{i_{m+1}}$ is a projective cover of $J e_{i_{m}}$ and $R e_{i_{1}}$ is a projective cover of $J e_{i_{k}}$; in particular, the only simple factors appearing in the composition series for the $R e_{i_{m}}$ are $S_{i_{1}}, S_{i_{2}}, \ldots, S_{i_{k}}$. Moreover, absence of candidates with projective dimension $\leqslant 1$ among the $S_{i_{m}}$ forces all the composition lengths $c_{i_{m}}$ to be equal.

THEOREM 6. Given a left serial ring $R$, the determinant of its Cartan matrix is 1 if and only if its left global dimension is finite. In any case the determinant is nonnegative.

Proof. We revert to the usual restrictions on $R$. If $\operatorname{lgl} \operatorname{dim} R<\infty$, then the combination of Lemmas 3-5 allows successive elimination of primitive idempotents corresponding to simple modules of projective dimension $\leqslant 1$ until we are left with one idempotent. But, in this situation, finite global dimension is tantamount to semisimplicity, whence $\operatorname{det} C=1$.

Now assume that $\operatorname{lgl} \operatorname{dim} R=\infty$. By induction on the number $n$ of primitive idempotents we will show that either $\operatorname{det} C=0$ or $\operatorname{det} C>1$. The case $n=1$ is trivial. For the induction step we may start with $n>1$ primitive idempotents $e_{i}$, none of which gives rise to a simple module $R e_{i} / J e_{i}$ of projective dimension $\leqslant 1$ (otherwise Lemmas 4 and 5 would permit us to discard one idempotent and invoke the induction hypothesis). That the quiver of $R$ is a tree is impossible, since its root would correspond to a projective module of length 1 . Therefore the quiver contains a cycle or loop with vertices $e_{1}, \ldots, e_{k}(k \geqslant 1)$, say. Since the composition series of $R e_{1}, \ldots, R e_{k}$ involve only the simple modules $S_{1}, \ldots, S_{k}$, the Cartan matrix of $R$ has block form

$$
\left[\begin{array}{cc}
C_{1} & X \\
0 & C_{2}
\end{array}\right],
$$

where $C_{1}$ is a $k \times k$ matrix as treated in Lemmas 1 and 2, whence $\operatorname{det} C_{1}=0$ or $\operatorname{det} C_{1}>1$. Note that $C_{2}$ is the Cartan matrix of $e R e$, where $e=1-e_{1}-\cdots-e_{k}$. If $1 \mathrm{gldim} e R e<\infty$, we have $\operatorname{det} C_{2}=1$ by the first part of the proof otherwise the induction hypothesis yields $\operatorname{det} C_{2} \geqslant 0$. In either case, $\operatorname{det} C=\left(\operatorname{det} C_{1}\right)\left(\operatorname{det} C_{2}\right)$ is either zero or greater than 1. 
COROllary 7. Let $R$ be an Artin algebra which is left or right serial, and let $C$ be its left Cartan matrix. Then $\operatorname{det} C=1$ if and only if $\mathrm{gl} \operatorname{dim} R<\infty$. In any case $\operatorname{det} C \geqslant 0$.

Proof. It suffices to note that in the case of an Artin algebra the left and right Cartan matrices have the same determinant. This follows from a straightforward modification of an argument due to Nakayama [12, Theorem 3].

3. Negative examples and positive remarks. For any left artinian ring, finite left global dimension narrows the range of possible values for $\operatorname{det} C$ to \pm 1 (see [2, Proposition 21; 13, Proposition 1.1]).

Our first two examples demonstrate that both +1 and -1 can also occur as Cartan determinants of artinian rings of infinite left global dimension. The examples we exhibit are both algebras over a field $F$ of Loewy length 2 and $F$-dimension 5 (one can easily convince oneself that $F$-dimension 5 is the lowest possible for either of these phenomena to occur). An example featuring $\operatorname{det} C=-1$ was already given by Eilenberg, Ikeda and Nakayama in [3]; however, their example is of dimension 12 over the base field.

EXAMPLE 8. Combining infinite global dimension with det $C=1$. Let $F$ be any field and $R$ the subring of $M_{4}(F)$ consisting of all matrices of the form

$$
\left[\begin{array}{cccc}
a & u & 0 & v \\
0 & b & 0 & 0 \\
0 & 0 & b & w \\
0 & 0 & 0 & a
\end{array}\right], \quad \text { where } a, b, u, v, w \in F
$$

$R$ has two primitive idempotents: $e_{1}=e_{11}+e_{44}$ and $e_{2}=e_{22}+e_{33}$. It is straightforward to check $J^{2}=0, e_{1} J e_{1} \neq 0, e_{2} J e_{1} \neq 0, e_{1} J e_{2} \neq 0$ and $e_{2} J e_{2}=0$, whence $J e_{1} \cong S_{1} \oplus S_{2}$ and $J e_{2} \cong S_{1}$ (again, we set $S_{i}=R e_{i} / J e_{i}$ ). It follows that $g 1 \operatorname{dim} R=$ $\infty$, whereas $C=\left[\begin{array}{ll}2 & 1 \\ 1 & 1\end{array}\right]$

EXAMPLE 9. Combining infinite global dimension with det $C=-1$. Starting again with a field $F$, this time we let $R$ be the subring of $M_{5}(F)$ consisting of all matrices of the form

$$
\left[\begin{array}{lllll}
a & 0 & u & v & 0 \\
0 & b & 0 & 0 & w \\
0 & 0 & b & 0 & 0 \\
0 & 0 & 0 & b & 0 \\
0 & 0 & 0 & 0 & a
\end{array}\right], \quad \text { where } a, b, u, v, w \in F
$$

Putting $e_{1}=e_{11}+e_{55}$ and $e_{2}=e_{22}+e_{44}$, we obtain $J e_{1} \cong S_{2}$ and $J e_{2} \cong S_{1} \oplus S_{1}$, whence $g l \operatorname{dim} R=\infty$ and $C=\left[\begin{array}{ll}1 & 2 \\ 1 & 1\end{array}\right]$.

Even though, for general artinian rings of Loewy length 2, the determinant of the Cartan matrix fails to distinguish between finite and infinite global dimension, the Cartan matrix as a whole still does. In fact, from a result of Jans and Nakayama [8, 
Proposition 10], it follows that, given a left artinian ring $R$ of Loewy length 2,

$$
1 \mathrm{gl} \operatorname{dim} R \leqslant k \Leftrightarrow(C-I)^{k+1}=0
$$

and

$$
1 \mathrm{gl} \operatorname{dim} R<\infty \Leftrightarrow(C-I)^{n}=0 ;
$$

here $I$ denotes the $n \times n$ identity matrix. We include a very short and elementary argument for a mild reinforcement of these equivalences.

REMARK 10. If $J^{2}=0$, the following statements are equivalent:

(a) $1 \mathrm{gl} \operatorname{dim} R<\infty$;

(b) $C$ is an upper triangular matrix with entries 1 along the main diagonal (for a suitable arrangement of the primitive idempotents).

In any case, the projective dimensions of the simple modules can be read off the columns of the Cartan matrix as follows: The columns containing a 1 in position $(j, j)$ and 0 elsewhere correspond to the simple projectives $R e_{j}$; say this occurs for the columns with indices $j$ in $D_{0}$. The columns of index $j \notin D_{0}$ containing a 1 in position $(j, j)$ and additional nonzero entries only in the positions $(l, j)$, with $l \in D_{0}$, correspond to the projectives $R e_{j}$ such that $\mathrm{p} \operatorname{dim} S_{j}=1$; say this is the case for the columns with indices $j$ in $D_{1}$. For, columns with indices $j \notin D_{0} \cup D_{1}$ containing a 1 in position $(j, j)$ and further nonzero entries only in the positions $(l, j)$, with $l \in D_{0} \cup D_{1}$, correspond to the projectives $R e_{j}$ such that p $\operatorname{dim} S_{j}=2$. When this procedure of successively filtering out the simple modules of projective dimension $0,1,2,3, \ldots$ comes to a halt, the leftover columns correspond to the projectives $R e_{j}$ such that $\mathrm{p} \operatorname{dim} S_{j}=\infty$.

Proof. $J^{2}=0$ implies $J e_{j}=S_{1}^{t_{j 1}} \oplus \cdots \oplus S_{n}^{t_{j n}}$ with $t_{j n} \geqslant 0$. Thus, p $\operatorname{dim} S_{j}=k<$ $\infty$ is tantamount to $t_{j j}=0$ and $\sup \left\{\mathrm{p} \operatorname{dim} S_{l}: t_{j l} \neq 0\right\}=k-1$. If we arrange the primitive idempotents such that $\mathrm{p} \operatorname{dim} S_{j} \leqslant \mathrm{p} \operatorname{dim} S_{j+1}$, the claimed equivalence follows immediately, and so does the supplementary statement.

Our final example settles two issues with one stroke. On the one hand, it shows that, among the left artinian rings of Loewy length 3, knowledge of the Cartan matrix no longer suffices to determine whether the left global dimension is finite. On the other hand, the first of the rings we construct has finite global dimension, but does not have any simple modules of projective dimension $\leqslant 1$. This contrasts with the rings we studied in $\S 2$, with the case $J^{2}=0$, and with the rings investigated by Zacharia [14].

EXAMPLE 11. Let $F$ be any field.

(a) Let $R$ be the subring of $M_{7}(F)$ consisting of all matrices of the form

$$
\left[\begin{array}{ccccccc}
c & 0 & x & u & w & m & q \\
0 & b & y & 0 & v & 0 & r \\
0 & 0 & a & 0 & z & 0 & s \\
0 & 0 & 0 & b & 0 & n & 0 \\
0 & 0 & 0 & 0 & b & 0 & 0 \\
0 & 0 & 0 & 0 & 0 & c & 0 \\
0 & 0 & 0 & 0 & 0 & 0 & c
\end{array}\right] .
$$


There are three primitive idempotents; $e_{1}=e_{33}, e_{2}=e_{22}+e_{44}+e_{55}, e_{3}=e_{11}+$ $e_{66}+e_{77}$, and the corresponding projective left modules $R e_{j}$ have the following structure: $J e_{1} \cong S_{2} \oplus S_{3}, J e_{2} \cong R e_{1} \oplus S_{3}, J e_{3}=R e_{1} \oplus L$ with $L / J L \cong S_{2}$ and $J L$ $\cong S_{3}$. From this information we derive projective resolutions of the simple modules
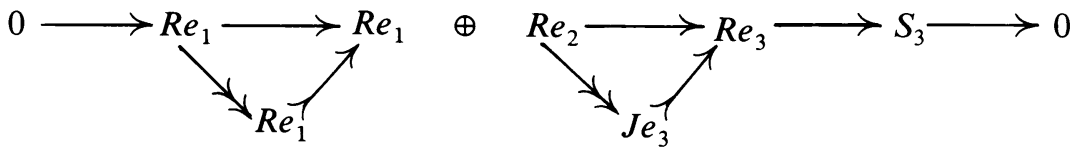

$\left(\mathrm{p} \operatorname{dim} S_{3}=2\right)$,

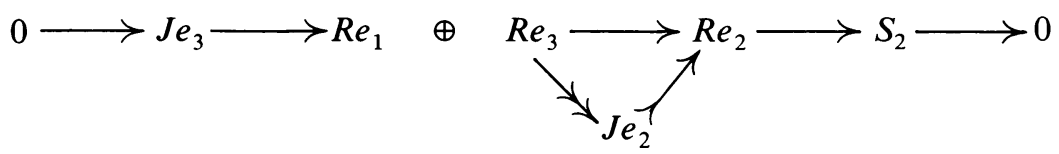

$\left(\mathrm{p} \operatorname{dim} S_{2}=3\right)$,

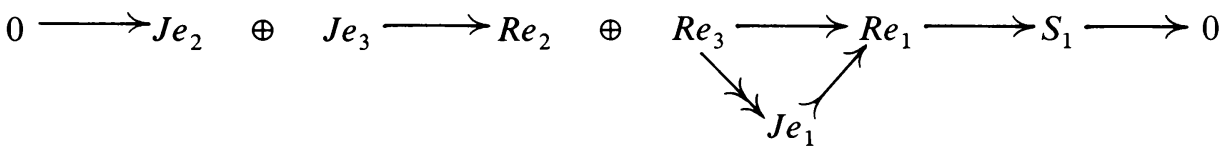

$\left(\mathrm{p} \operatorname{dim} S_{1}=4\right)$.

In particular, we see that $\mathrm{gl} \operatorname{dim} R=4$. Moreover, counting composition factors yields the Cartan matrix

$$
C=\left[\begin{array}{lll}
1 & 1 & 1 \\
1 & 2 & 2 \\
1 & 2 & 3
\end{array}\right] .
$$

(b) Modifying $R$ by moving the entry $n$ from position $(4,6)$ to position $(2,6)$, we arrive at a ring $\tilde{R}$ with gl $\operatorname{dim} \tilde{R}=\infty$ (since $\tilde{S}_{3}$ is isomorphic to a direct summand of $\left.\tilde{J} e_{3}\right)$ that has the same Cartan matrix as $R$.

\section{REFERENCES}

1. P. J. Davis, Circulant matrices, Wiley, New York, 1979.

2. S. Eilenberg, Algebras of cohomologically finite dimension, Comment. Math. Helv. 28 (1954), 310-319.

3. S. Eilenberg, M. Ikeda and T. Nakayama, On the dimension of modules and algebras. I, Nagoya Math. J. 8 (1955), 49-57.

4. K. R. Fuller, Generalized uniserial rings and their Kupisch series, Math. Z. 106 (1968), 248-260.

5. K. R. Fuller and J. Haack, Rings with quivers that are trees, Pacific J. Math. 76 (1978), 371-379.

6. R. Gordon and E. L. Green, Modules with cores and amalgamation of indecomposable modules, Mem. Amer. Math. Soc. 187 (1977).

7. W. H. Gustafson, Global dimension in serial rings, 1983 (typescript).

8. J. P. Jans and T. Nakayama, On the dimension of modules and algebras. VII, Nagoya Math. J. 11 (1957), 67-76.

9. G. J. Janusz, Some left serial algebras of finite type, J. Algebra 23 (1972), 404-411.

10. H. Kupisch, Beiträge zur Theorie nichthalbeinfacher Ringe mit Minimalbedingung, J. Reine Angew. Math. 201 (1959), 100-112.

11. I. Murase, On the structure of generalized uniserial rings. I, II, III, Sci. Papers College Gen. Ed. Univ. Tokyo 13 (1963), 1-22; ibid. 13 (1963), 131-158; ibid. 14 (1964), 11-25. 
12. T. Nakayama, Some studies on regular representations, induced representations and modular representations, Ann. of Math. (2) 39 (1938), 361-369.

13. G. V. Wilson, The Cartan map on categories of graded modules, J. Algebra 85 (1983), 390-398.

14. D. Zacharia, On the Cartan matrix of an Artin algebra of global dimension two, J. Algebra 82 (1983), 353-357.

Department of Mathematics, University of Ottawa, Ottawa, Canada K1N 984

Department of Mathematics, University of Iowa, Iowa City, IOWA 52242

Department of Zoology, University of Iowa, Iowa City, Iowa 52242

Department of Mathematics, Universität Passau, 8390 Passau, W. Germany 